\title{
Endoscopic endonasal clip ligation of cerebral aneurysms: an anatomical feasibility study and future directions
}

\author{
Oszkar Szentirmai, MD, ${ }^{1}$ Yuan Hong, MD, ${ }^{1,6}$ Lino Mascarenhas, MD, ${ }^{1}$ Al Amin Salek, MD, ${ }^{1}$ \\ Philip E. Stieg, PhD, MD, ${ }^{1}$ Vijay K. Anand, MD, ${ }^{2}$ Aaron A. Cohen-Gadol, MD, MSc, ${ }^{4,5}$ and \\ Theodore H. Schwartz, MD'-3
}

Departments of ${ }^{1}$ Neurosurgery, ${ }^{2}$ Otolaryngology, and ${ }^{3}$ Neuroscience, Weill Cornell Medical College, NewYork-Presbyterian Hospital, New York, New York; ${ }^{4}$ Goodman Campbell Brain and Spine and Indiana University Department of Neurological Surgery; ${ }^{5}$ Indiana University Simon Cancer Center, Indianapolis, Indiana; and ${ }^{6}$ Department of Neurosurgery, Second Affiliated Hospital of Zhejiang University School of Medicine, Hangzhou, China

\begin{abstract}
OBJECTIVE The expansion of endovascular procedures for obliteration of cerebral aneurysms highlights one of the drawbacks of clip ligation through the transcranial route, namely brain retraction or brain transgression. Sporadic case reports have emerged over the past 10 years describing endonasal endoscopic clip ligation of cerebral aneurysms. The authors present a detailed anatomical study to evaluate the feasibility of an endoscopic endonasal approach for application of aneurysm clips.
\end{abstract}

METHODS Nine human cadaveric head specimens were used to evaluate operative exposures for clip ligation of aneurysms in feasible anterior and posterior circulation locations. Measurements of trajectories were completed using a navigation system to calculate skull base craniectomy size, corridor space, and the surgeon's ability to gain proximal and distal control of parent vessels.

RESULTS In each of the 9 cadaveric heads, excellent exposure of the target vessels was achieved. The transplanum, transtuberculum, and transcavernous approaches were used to explore the feasibility of anterior circulation access. Application of aneurysm clips was readily possible to the ophthalmic artery, $A_{1}$ and $A_{2}$ segments of the anterior cerebral artery, anterior communicating artery complex, and the paraclinoid and paraclival internal carotid artery. The transclival approach was explored, and clips were successfully deployed along the proximal branches of the vertebrobasilar system and basilar trunk and bifurcation. The median sizes of skull base craniectomy necessary for exposure of the anterior communicating artery complex and basilar tip were $3.24 \mathrm{~cm}^{2}$ and $4.62 \mathrm{~cm}^{2}$, respectively. The mean angles of surgical corridors to the anterior communicating artery complex and basilar tip were $11.4^{\circ}$ and $14^{\circ}$, respectively. Although clip placement was feasible on the basilar artery and its branches, the associated perforating arteries were difficult to visualize, posing unexpected difficulty for safe clip application, with the exception of ventrolateral-pointing aneurysms.

CONCLUSIONS The authors characterize the feasibility of endonasal endoscopic clip ligation of aneurysms involving the paraclinoid, anterior communicating, and basilar arteries and proximal control of the paraclival internal carotid artery. The endoscopic approach should be initially considered for nonruptured aneurysms involving the paraclinoid and anterior communicating arteries, as well as ventrolateral basilar trunk aneurysms. Clinical experience will be mandatory to determine the applicability of this approach in practice.

http://thejns.org/doi/abs/10.3171/2015.1.JNS142650

KEY WORDS endoscopic; endonasal; cerebral aneurysm; clip ligation; anatomy; surgical technique

$\mathrm{T}$ HE field of vascular neurosurgery is continually evolving as a result of the ongoing search for more effective and safer treatments. Following decades of microsurgical clip ligation, endovascular procedures have emerged as a viable alternative, and for certain patients, they are a superior option.
Endoscopic endonasal surgery is still evolving as a routine alternative to transcranial approaches for treatment of skull base tumors. ${ }^{11}$ The introduction of the endoscopic endonasal approach for treatment of cerebral aneurysms is the latest evolutionary and innovative step in refining endoscopic surgery; this technique has been reported only in 
a handful of case reports in the literature. ${ }^{1-4,6-10}$ No study to date has explored the indications and potential applications of the endonasal route for managing intracranial aneurysms. Therefore, a more in-depth study of vascular anatomy exposed through the transnasal route and relevant techniques in the application of aneurysm clips using endoscopy was undertaken.

\section{Methods}

Nine formalin-fixed, silicon-injected human cadaveric heads were used for this study. All specimens were placed in a Mayfield head holder. The Storz endoscopy system (Karl Storz) with a Hopkins rigid $0^{\circ}, 30^{\circ}$, and $45^{\circ}$ endoscope or a Visionsense 3D and 3DHD prototype endoscope (Petach-Tikva) were used with a Storz flexible endoscope holder. All dissections were performed through the endonasal corridor.

The $0^{\circ}$ and $30^{\circ}$ endoscopes were used for dissections, and $45^{\circ}$ endoscopes were used for examination of clip blades following clip application. All 9 specimens were dissected for exposure of the cerebral vasculature of interest, with removal of the surrounding bone and dura for mimicking the operative approach.

In all specimens, the area of the skull base craniectomy was measured for both the transtuberculum/transplanum and the transclival approaches. The transcavernous approach was used to expose the paraclival internal carotid artery (ICA) and the proximal ophthalmic artery. Bone drilling was performed using a high-speed drill. Aesculap provided titanium aneurysm clips and appliers. Fiducial markers were sutured to the cadavers; scalp and thin-cut head CT scans were obtained. Segmentation and registration was completed on BrainLab Cranial Planning software. Navigation was then used to localize and quantitate craniectomy size under endoscopic guidance. Maximal surgical corridors were determined using BrainLab navigation software and corridor angles were calculated for each specimen

\section{Results}

The transplanum and transtuberculum approaches exposed the proximal $A_{1}$ and $A_{2}$ segments of the anterior cerebral artery and the anterior communicating artery (ACoA) (Fig. 1). This route allowed clip ligation of the distal $\mathrm{A}_{1}$ branches, $\mathrm{ACoA}$, proximal $\mathrm{A}_{2}$ branches to the level of the pericallosal segment (Fig. 2). Proximal and distal control was most readily possible at the level of the ACoA complex (Fig. 3). An anterosuperiorly projecting aneurysm dome would offer the most favorable access for endonasal clip application, best visualization of the aneurysm neck as well as perforators and minimal risk of sac rupture. The addition of the transcavernous approach also exposed the paraclival and paraclinoid ICA territories and secured proximal vascular control (Fig. 4). The clinoidal ICA was found where it entered the subarachnoid space and its circumferential dissection secured proximal control. Using this approach, medially located ophthalmic or hypophyseal artery aneurysms were very reasonable candidates for endonasal clip ligation (Fig. 5).

Following clip placement at the level of the paraclinoid

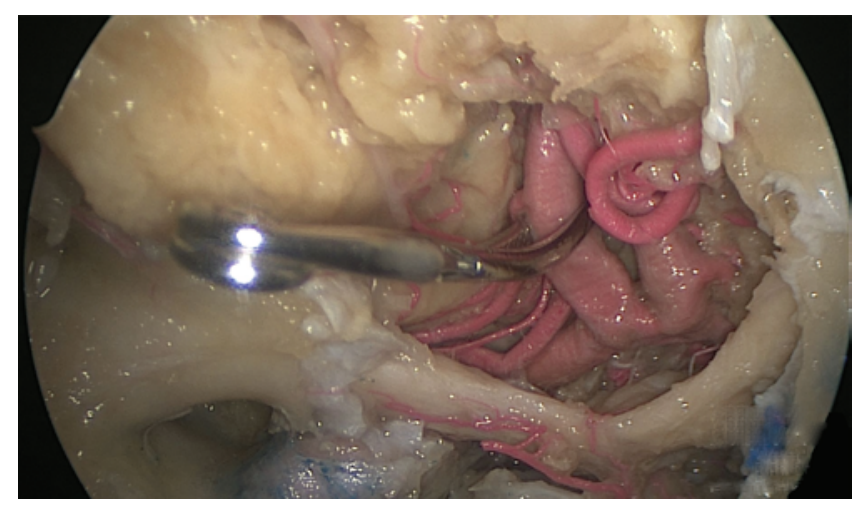

FIG. 1. Transtuberculum, transplanum exposure of the ACoA complex with a curved aneurysm clip placed on the distal right $A_{1}$. Figure is available in color online only.

ICA and ACoA complex, the proximity of the anterosuperiorly or laterally projecting clips to the durotomy allowed multilayer closure between the sella and the planum with the aneurysm clip resting parallel to the floor of the anterior cranial fossa.

The transclival route allowed deployment of aneurysm clips on the distal vertebral artery, proximal anterior and posterior cerebellar arteries, basilar artery and basilar bifurcation, as well as the proximal superior cerebellar artery, posterior cerebral artery and proximal posterior communicating artery (Fig. 6). The use of scopes at different angles provided additional views to ensure optimal placement of the clips. Ventrolateral-pointing basilar trunk aneurysms were considered good candidates for clip ligation because proximal control was readily available and angled endoscopes afforded panoramic visualization of the basilar perforators.

After clip deployment at the level of the distal basilar artery, depending on the relationship of the basilar bifurcation to the sellar floor, an anteriorly projecting aneurysm clip may be incorporated into the multilayer closure. When choosing an aneurysm clip, the operator should consider a clip configuration that will rest the hinge of the clip adjacent and preferably parallel to the floor of the sella, therefore allowing an inlay dural closure. An angled or bayoneted aneurysm clip will facilitate direct visualiza-

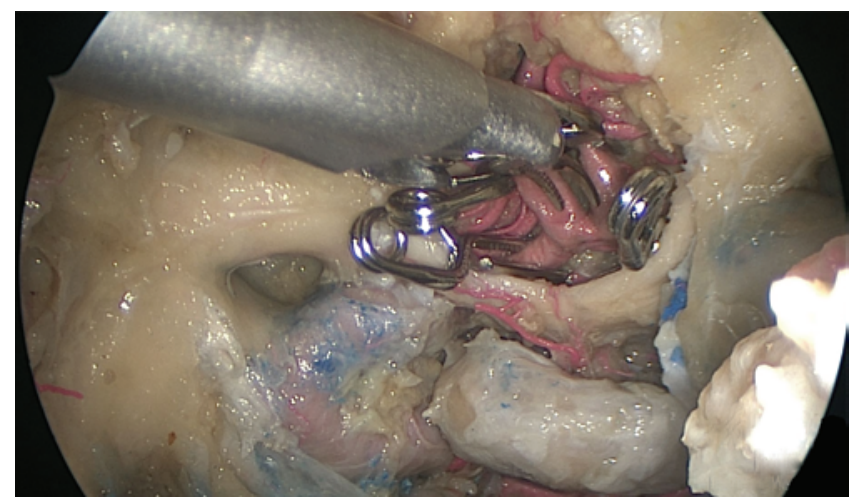

FIG. 2. Transtuberculum, transplanum exposure with temporary clips placed on bilateral $A_{1}$ and $A_{2}$ segments for proximal and distal control with the fifth clip being applied to the ACoA. Figure is available in color online only. 


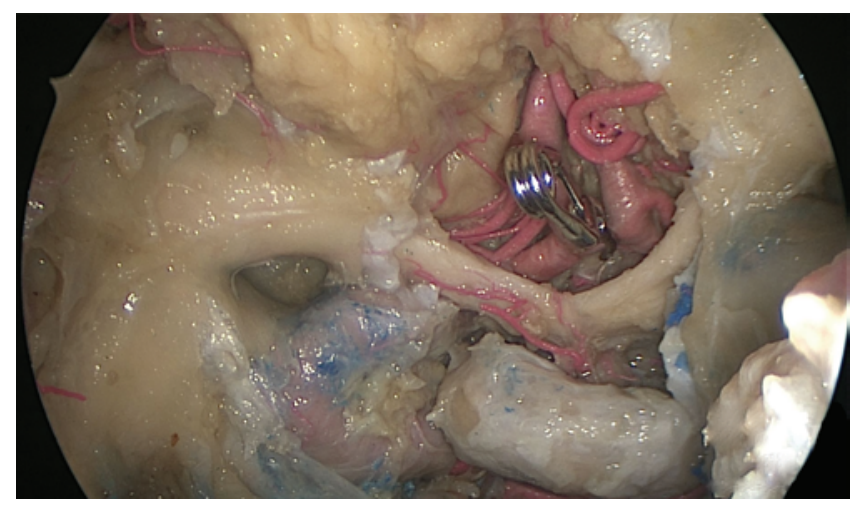

FIG. 3. After application of the ACoA aneurysm clip, the 4 temporary clips were removed. Figure is available in color online only.

tion of the clip blades, not only in the anterior-posterior, but also the medial-lateral planes. This maneuver will also make the multilayer closure easier by placing the clip along the lateral wall of the craniectomy.

The median craniectomy sizes for exposure of the paraclinoid ICA/ACoA complex and basilar bifurcation regions were $3.24 \mathrm{~cm}^{2}$ and $4.62 \mathrm{~cm}^{2}$, respectively. This area of exposure was sufficient in all cases for placement of 2-3 clips, including the application of 1-2 temporary clips for proximal vascular control on the ICA, basilar, and $\mathrm{A}_{1}$ arteries and a permanent clip on the virtual aneurysm.

Maximal operative corridor angles were calculated with the assistance of neuronavigation (Fig. 7). For the ICA/ACoA and basilar bifurcation targets, the mean corridor angles were $11.4^{\circ}$ and $14^{\circ}$, respectively. This transnasal route provided generous space for placement of a 4 $\mathrm{mm}$ endoscope, 2 large bore suction tips (or 1 suction and 1 dissector) and a clip applier.

\section{Discussion}

Innovative surgical approaches for vascular lesions

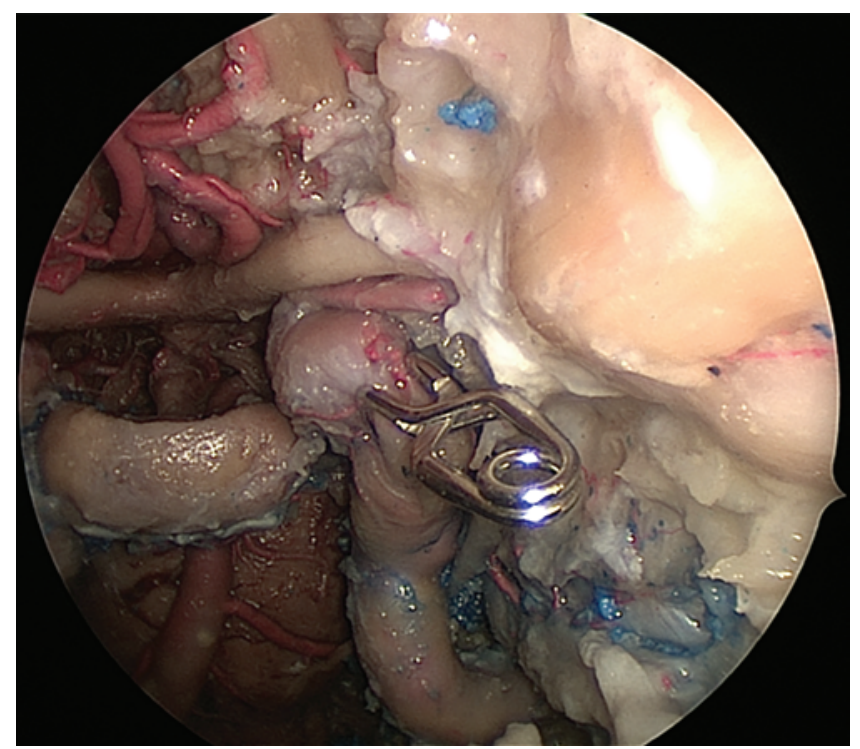

FIG. 4. Transcavernous approach permits proximal clips on the cavernous ICA. Figure is available in color online only.

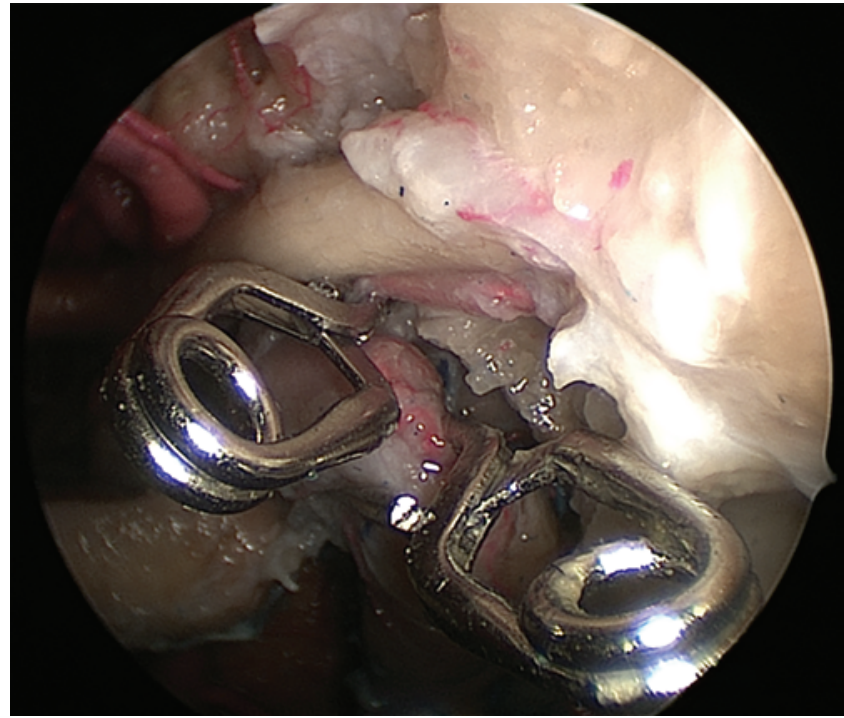

FIG. 5. Transcavernous approach also can be used to secure the proximal ophthalmic artery. Figure is available in color online only.

including clip ligation of cerebral aneurysms or resection of arteriovenous malformations have been recently performed using the endoscopic endonasal approach and published in 9 case reports. ${ }^{1-4,6-10}$ These case reports are based on clip ligation of 2 ACoA, 2 basilar artery, 2 vertebral artery, 1 ophthalmic artery, 1 paraclinoid ICA, and 1 superior hypophyseal artery aneurysms and 1 clival arteriovenous malformation (Table 1). Three of these cases involved ruptured aneurysms. None of these cases was performed in a hybrid endovascular operating room suite. There was no mortality among these patients. These reports have further complemented our findings regarding the feasibility of aneurysm clip ligation through the endonasal endoscopic route.

Patients harboring brain aneurysms frequently manifest unusual vascular anatomy, predisposing them to aneurysm formation. Our study is limited by its design to explore only anatomical relationships in cadavers with no history of aneurysms. Therefore, the present study is only the first step in assessing the feasibility of aneurysm clip

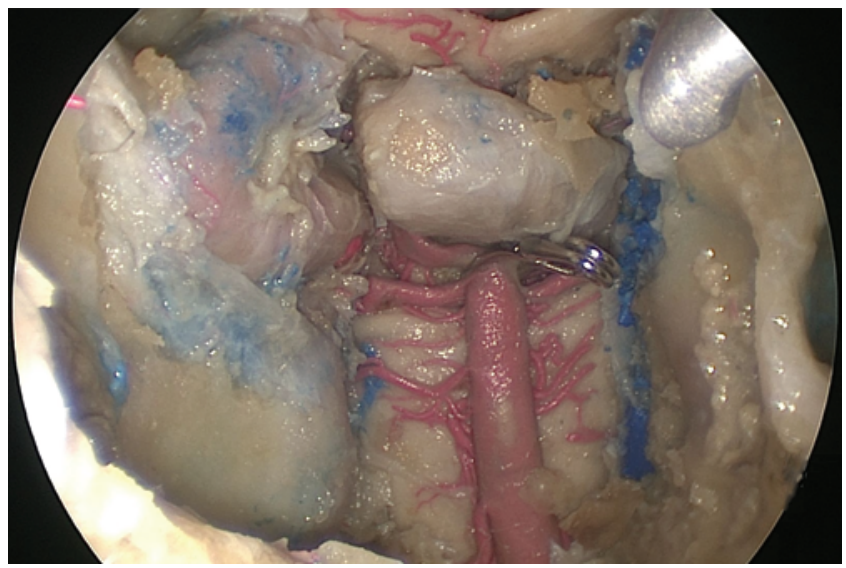

FIG. 6. Transclival approach with placement of an aneurysm clip at the basilar artery between the superior cerebellar and posterior cerebral arteries. Figure is available in color online only. 


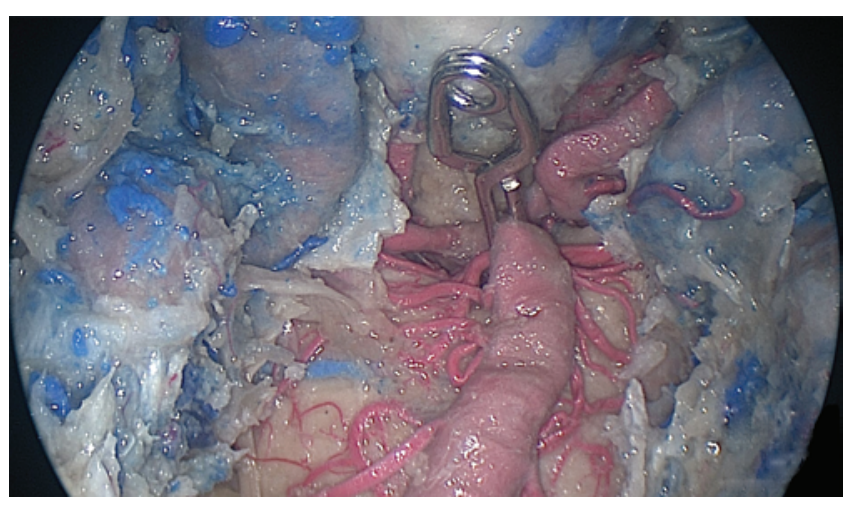

FIG. 7. View from below with a $30^{\circ}$ scope allows additional visualization of the perforators and the placement of the clip. Figure is available in color online only.

ligation through the endoscopic endonasal route. Patient series are needed to clarify the safety of the endoscopic endonasal corridor for clip ligation of aneurysms and especially for ruptured cases.

We plan to focus our initial attempts in the operating room to explore this technique for patients with small unruptured aneurysms and favorable anatomy (Fig. 8). Favorable anatomy should preclude any significant risk to the aneurysm dome during the dural opening (inferiorly pointing ACoA aneurysms) and allow direct visualization of the aneurysm neck as well as generous control over proximal and distal vessels (ventrolateral basilar trunk, medial paraclinoid, and anterosuperiorly pointing ACoA aneurysms). Ventrolateral basilar trunk and medially pointing proximal ICA aneurysms are often difficult to expose through the transcranial route, and the endonasal route can be an effective alternative. The endonasal exposure should also facilitate dissection of vessels away from the aneurysm neck or dome and allow dissection around the dome, if needed. The available endonasal working axes and angles expanded by the angled endoscopes offer a special opportunity for visualizing perforating arteries in the regions deemed difficult to see through the transcranial corridors.

It is also important for the endonasal route to allow for clip repositioning. Often, the hinge of the clip moves upon its release from the clip applier, making its reengagement within the clip applier a technically difficult task due to the limited working angles provided by this route. Therefore, design of new clips that can engage within their appliers through expanded working angles is necessary. Based on our study, we believe that paraclinoid and anterosuperiorly pointing ACoA aneurysms should be among the first types of aneurysms considered for treatment via the endonasal corridor.

Meanwhile, to maximize patient safety, the procedure should be preferentially performed in a hybrid endovascular operating room suite by teams experienced in transcranial and endovascular aneurysm surgery and endoscopic skull base surgery. Dural opening over an aneurysm where the dome partially obstructs the view or access to the aneurysm neck would carry a high risk of intraoperative rupture without proximal control. This difficult and lifethreatening situation may be controlled with temporary

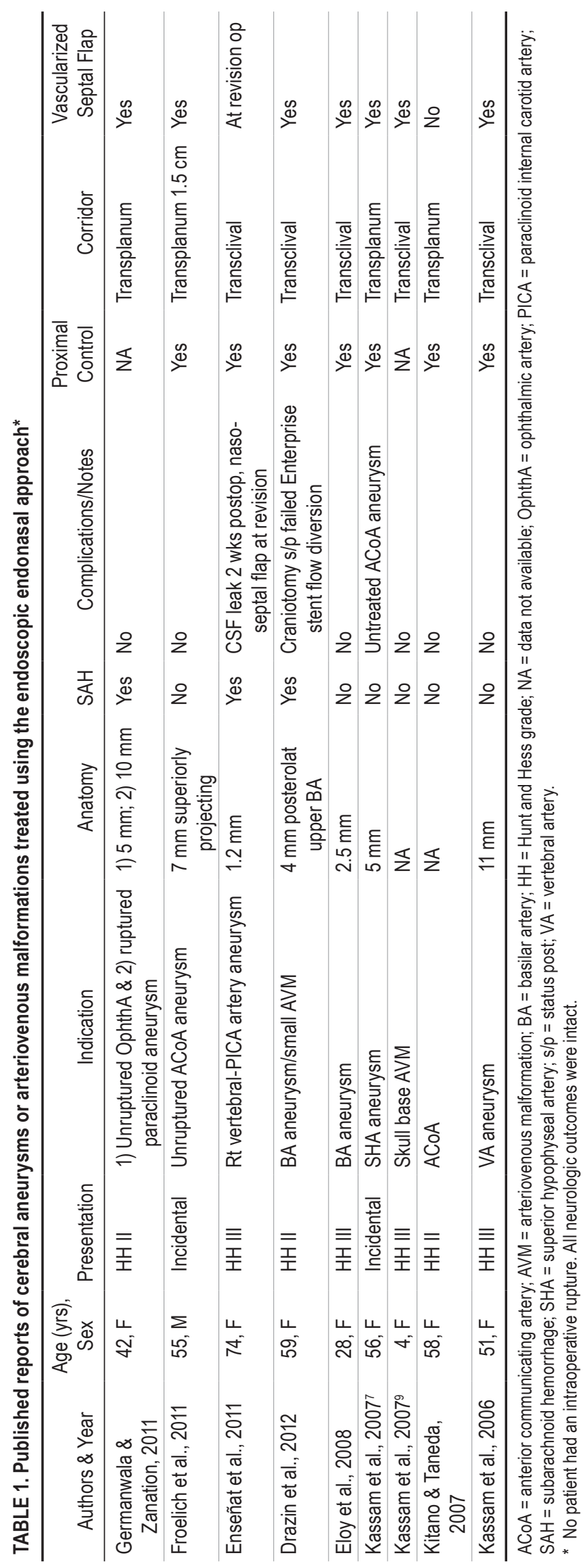




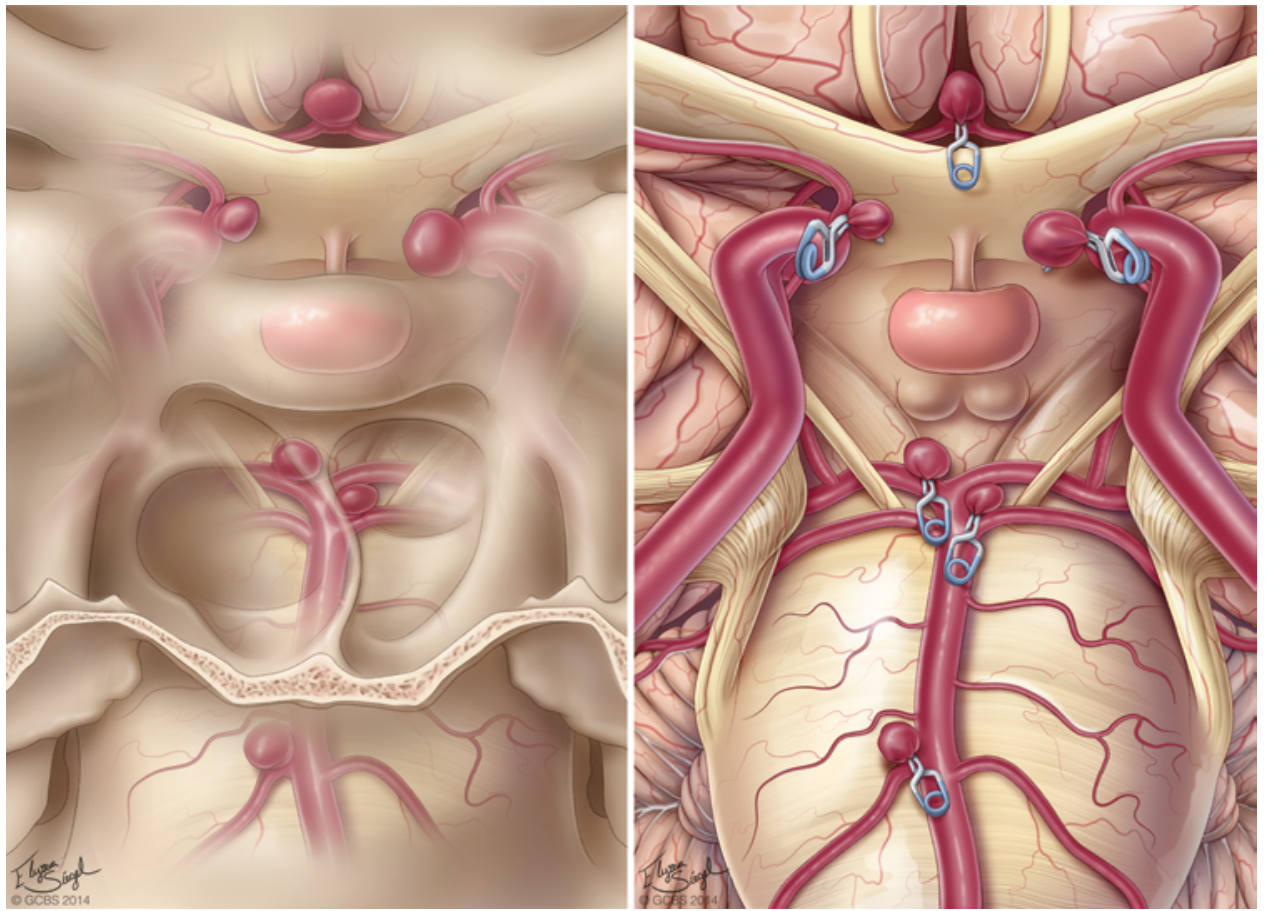

FIG. 8. Panoramic illustrations demonstrating the location (left) and clip configurations (right) through the endonasal route. Copyright Goodman Campbell Brain and Spine, 2014. Published with permission. Figure is available in color online only.

adenosine-induced cardiac arrest or by using temporary endovascular or surgical parent vessel occlusion. Therefore, the team should be prepared to expose the carotid artery at the neck or use adenosine-induced cardiac arrest by placement of percutaneous pacers. Further development of endoscopic vascular instrumentation including clip appliers is critical to secure unobstructed operative working angles in the event of an intraoperative aneurysm rupture. Reconstruction of the craniectomy is of paramount importance to prevent postoperative CSF leaks and meningitis, and incorporation of the aneurysm clip into the multilayer closure requires preplanning at the time of clip selection and placement. ${ }^{5}$ Adequate skull base reconstruction in the presence of a protruding clip will be challenging and alternative low-profile clips may be needed to minimize the risk of postoperative CSF fistula formation. Coverage of the clip by fat graft to avoid its erosion through the dural reconstruction is an alternative option. Most importantly, if the endoscopic route does not satisfy the basic principles of aneurysm surgery (that is, proximal and distal parent vessel control, etc.) upon intraoperative inspection, then the procedure should be aborted and alternative traditional transcranial treatment options should be employed.

Finally, with recent progressive advances in endovascular neurosurgery, fewer aneurysms require microsurgical clip ligation. Of patients with aneurysms requiring microsurgical clip ligation, only a very small proportion are best suited for endoscopic surgery. Therefore, the indications for endoscopic clip ligation of aneurysms are limited, but this technique should be available in the armamentarium of cerebrovascular and endoscopic skull base surgeons when needed. Although endovascular techniques continue to advance, it is unlikely that the endoscopic route will be widely used for aneurysm clip ligation since only very select cases are appropriate for this methodology.

\section{Conclusions}

Endonasal endoscopic aneurysm clip ligation has the potential to emerge as a safe, effective, and advantageous alternative to transcranial and endovascular options for a small percentage of patients. Aneurysms of the paraclinoid ICA and ACoA complex with supramedial and anterosuperior projections, respectively, would offer the most favorable endoscopic endonasal anatomy. Ventrolateral basilar trunk aneurysms may also be appropriate. Caution must be exercised during the initial clinical applications to ensure reliable proximal and distal vascular control and to improve upon current endoscopic microsurgical instrumentation. Patient safety during an intraoperative rupture is most important. Optimal results might be best achieved if these endoscopic surgeries are performed in a hybrid operating room with endovascular capabilities by experienced endoscopic skull base and transcranial vascular neurosurgeons.

Endoscopic transnasal aneurysm clip ligation will not replace transcranial microsurgical techniques but can be used as part of the multimodality management of intracranial aneurysms in very select cases. In this first cadaver series we explore the feasibility of this approach. Future clinical cases will be required to determine the applicability of this approach in practice.

\section{References}

1. Drazin D, Zhuang L, Schievink WI, Mamelak AN: Expand- 
ed endonasal approach for the clipping of a ruptured basilar aneurysm and feeding artery to a cerebellar arteriovenous malformation. J Clin Neurosci 19:144-148, 2012

2. Eloy JA, Carai A, Patel AB, Genden EM, Bederson JB: Combined endoscope-assisted transclival clipping and endovascular stenting of a basilar trunk aneurysm: case report. Neurosurgery 62 (3 Suppl 1):142-144, 2008

3. Enseñat J, Alobid I, de Notaris M, Sanchez M, Valero R, Prats-Galino A, et al: Endoscopic endonasal clipping of a ruptured vertebral-posterior inferior cerebellar artery aneurysm: technical case report. Neurosurgery 69 (1 Suppl Operative):E121-E128, 2011

4. Froelich S, Cebula H, Debry C, Boyer P: Anterior communicating artery aneurysm clipped via an endoscopic endonasal approach: technical note. Neurosurgery 68 (2 Suppl Operative):310-316, 2011

5. Garcia-Navarro V, Anand VK, Schwartz TH: Gasket seal closure for extended endonasal endoscopic skull base surgery: efficacy in a large case series. World Neurosurg 80:563-568, 2013

6. Germanwala AV, Zanation AM: Endoscopic endonasal approach for clipping of ruptured and unruptured paraclinoid cerebral aneurysms: case report. Neurosurgery 68 (1 Suppl Operative):234-240, 2011

7. Kassam AB, Gardner PA, Mintz A, Snyderman CH, Carrau RL, Horowitz M: Endoscopic endonasal clipping of an unsecured superior hypophyseal artery aneurysm. Technical note. J Neurosurg 107:1047-1052, 2007

8. Kassam AB, Mintz AH, Gardner PA, Horowitz MB. Carrau $\mathrm{RL}$, Snyderman CH: The expanded endonasal approach for an endoscopic transnasal clipping and aneurysmorrhaphy of a large vertebral artery aneurysm: technical case report. Neurosurgery 59:ONSE162-ONSE165, 2006

9. Kassam AB, Thomas AJ, Zimmer LA, Snyderman CH, Carrau RL, Mintz A, et al: Expanded endonasal approach: a fully endoscopic completely transnasal resection of a skull base arteriovenous malformation. Childs Nerv Syst 23:491-498, 2007

10. Kitano M, Taneda M: Extended transsphenoidal approach to anterior communicating artery aneurysm: aneurysm incidentally identified during macroadenoma resection: technical case report. Neurosurgery 61:E299-E300, 2007

11. Komotar RJ, Starke RM, Raper DM, Anand VK, Schwartz TH: Endoscopic skull base surgery: a comprehensive comparison with open transcranial approaches. Br J Neurosurg 26:637-648, 2012

\section{Author Contributions}

Conception and design: all authors. Acquisition of data: all authors. Analysis and interpretation of data: all authors. Drafting the article: all authors. Critically revising the article: all authors. Reviewed submitted version of manuscript: all authors. Approved the final version of the manuscript on behalf of all authors: Schwartz.

\section{Correspondence}

Theodore H. Schwartz, Department of Neurosurgery, Weill Cornell Medical Center, New York-Presbyterian Hospital, 525 E. 68th St., New York, NY 10021. email: schwarh@med.cornell.edu. 